\title{
MODELING NON STATIONARY HIDDEN SEMI-MARKOV CHAINS WITH TRIPLET MARKOV CHAINS AND THEORY OF EVIDENCE
}

\author{
Wojciech Pieczynski
}

\author{
INT/GET, Département CITI, CNRS UMR 5157 \\ 9, rue Charles Fourier, 91000 Evry, France
}

\begin{abstract}
Hidden Markov chains, enabling one to recover the hidden process even for very large size, are widely used in various problems. On the one hand, it has been recently established that when the hidden chain is not stationary, the use of the theory of evidence is equivalent to consider a triplet Markov chain and can improve the efficiency of unsupervised segmentation. On the other hand, hidden semi-Markov chains can also be considered as particular triplet Markov chains. The aim of this paper is to use these two points simultaneously. Considering a non stationary hidden semi-Markov chain, we show that it is possible to consider two auxiliary random chains in such a way that unsupervised segmentation of non stationary hidden semiMarkov chains is workable.
\end{abstract}

\section{INTRODUCTION}

Let $Z=(X, Y)$, with $X=\left(X_{1}, \ldots, X_{n}\right), Y=\left(Y_{1}, \ldots, Y_{n}\right)$ be two random chains, where each $X_{i}$ takes its values in $\Omega=\left\{\omega_{1}, \ldots, \omega_{k}\right\}$ and each $Y_{i}$ takes its values in $R$. We will say that $Z=(X, Y)$ is a classical hidden Markov chain with independent noise (HMC-IN) if its distribution reads $\quad p(z)=p\left(x_{1}\right) p\left(x_{2} \mid x_{1}\right) \ldots p\left(x_{n} \mid x_{n-1}\right) p\left(y_{1} \mid x_{1}\right) \ldots p\left(y_{n} \mid x_{n}\right)$. Then the hidden chain $X$ is a Markov one, and we add "independent noise" because the random variables $Y_{1}, \ldots$, $Y_{n}$ are independent conditionally on $X$. This condition can be relaxed and there exist hidden Markov models in which $X$ is Markovian and the random variables $Y_{1}, \ldots$, $Y_{n}$ are not independent conditionally on $X$; such a model will be called hidden Markov chains (HMC).

HMC-IN are widely used because unknown realisations of $X$ can be estimated from observed realisations of $Y$ by different Bayesian methods, even for very large values of $n$. Furthermore, different model parameters estimation methods, like the "expectation-maximization" (EM) algorithm or the "iterative conditional estimation" (ICE) one, are available, which enables unsupervised estimation of $X$ from $Y$.

Classically, HMC-IN have been extended in two directions:

(i) In HMC-IN the hidden chain $X$ is a Markov one, and thus the sojourn duration distribution in each state is exponential. In hidden semi-Markov chains with independent noise (HSMC-IN), which form an extension of HMC-IN, this distribution is of any kind. HSMC-IN are useful in many situations, as images sequence analysis [5], speech processing [6], or still tracking problems [15], among others;

(ii) more recently, HMC-IN have been extended to "pairwise Markov chains" (PMC [9]), in which one directly assumes the Markovianity of $Z=(X, Y)$ and in which $X$ is no longer necessarily a Markov chain, and to "triplet Markov chains" (TMC [10, 13]), in which one introduces a third auxiliary random chain $U=\left(U_{1}, \ldots, U_{n}\right)$ and assumes the Markovianity of the triplet $T=(X, U, Y)$. When the random variables $U_{1}, \ldots, U_{n}$ take their values in a discrete finite space, both PMC and TMC still enable to estimate $X$ from $Y$ by Bayesian methods. Let us mention that TMC can be also used when the three chains $X, U$, and $Y$ are continuous; in this case Bayesian segmentation methods are replaced by Kalman, or particle, filtering techniques [1, 4].

Otherwise, we have two following recent results:

(iii) let us return to classical HMC-IN, with unknown parameters and with a non stationary process $X$. It is possible to estimate the model parameters by EM or ICE; however, such estimation methods assume the stationarity of $X$ and would thus necessarily give wrong results, which in turn can imply poor restoration results of $X=x$. It then has been shown that replacing the non stationary priors by "evidential" stationary ones ("evidential" refers to the theory of evidence $[2,14]$ ) enables to improve the final segmentation results [7, 8]. Moreover, introducing such "evidential" priors is identical, from the mathematical viewpoint, to consider a particular TMC $[7,8]$;

(iv) very recently it has been showed that HSMC-IN can be seen as particular TMC $[11,13]$. 
The aim of this paper is to use points (iii) and (iv) above simultaneously. Considering a non stationary hidden semiMarkov chain, we introduce a TMC in which the auxiliary chain has two components. The first one models the HSMC-IN, as in [11], and the second one enables us to take into account, via evidential priors, its non stationarity, as in [7, 8]. We detail the use of such a model in Byayesian segmentation and briefly describe both EM and ICE parameter estimation methods.

\section{HIDDEN SEMI MARKOV CHAIN AS A TRIPLET MARKOV CAHINNTRODUCTION}

Since [11] is under submission and the problem is just mentioned in [13], let us briefly specify, via an example, why each HSMC-IN is a particular TMC. Let $(X, Y)$ be an HSMC-IN with the distribution given by :

(a) the distribution of $X_{1}$ on $\Omega$, denoted by $p\left(x_{1}\right)$;

(b) the transitions $\left(p\left(x_{i} \mid x_{i-1}\right)\right)_{i \geq 2}$ verifying $p\left(x_{i} \mid x_{i-1}\right)=0$ for $x_{i}=x_{i-1}$;

(c) $q$ a probability distribution on the set of natural numbers $N$, and

(d) distributions $p\left(y_{i} \mid x_{i}\right)$ on $R$.

Otherwise, let us consider a general TMC $T=(X, U, Y)$, with each $U_{i}$ taking its values in $N^{*}=N-\{0\}$. The general form if its distribution is then defined by $p\left(t_{1}\right)=p\left(x_{1}, u_{1}, y_{1}\right) \quad$ and the transitions $p\left(t_{n+1} \mid t_{n}\right)=p\left(x_{n+1}, u_{n+1}, y_{n+1} \mid x_{n}, u_{n}, y_{n}\right)$. These transitions can be detailed in different ways; in the following, we will consider them as being written

$$
\begin{aligned}
p\left(x_{n+1}, u_{n+1}, y_{n+1} \mid x_{n}, u_{n}, y_{n}\right)= & \\
& p\left(x_{n+1} \mid x_{n}, u_{n}, y_{n}\right) \times \\
& p\left(u_{n+1} \mid x_{n}, u_{n}, y_{n}, x_{n+1}\right) \times \\
& p\left(y_{n+1} \mid x_{n}, u_{n}, y_{n}, x_{n+1}, u_{n+1}\right)
\end{aligned}
$$

Then HSMC-IN defined by (a)-(d) can be seen as a TMC defined by $p\left(t_{1}\right)=p\left(x_{1}, u_{1}, y_{1}\right)$ and $(1)$, where $(d$ is the Dirac's mass):

$$
\begin{aligned}
& p\left(x_{n+1} \mid x_{n}, u_{n}, y_{n}\right)=p\left(x_{n+1} \mid x_{n}, u_{n}\right)=d\left(x_{n}\right) \quad \text { if } \quad u_{n}>1, \quad \text { and } \\
& p\left(x_{n+1} \mid x_{n}\right) \text { if } u_{n}=1 \\
& p\left(u_{n+1} \mid x_{n+1}, x_{n}, u_{n}, y_{n}\right)=p\left(u_{n+1} \mid u_{n}, x_{n}\right)=d\left(u_{n}-1\right) \text { if } u_{n}>1 \\
& \text { and } q\left(u_{n+1}\right) \text { if } u_{n}=1
\end{aligned}
$$

$$
p\left(y_{n+1} \mid x_{n}, u_{n}, y_{n}, u_{n+1}, x_{n+1}\right)=p\left(y_{n+1} \mid x_{n+1}\right) .
$$

Finally, we have a particular TMC where (1) reduces to

$$
\begin{aligned}
& p\left(x_{n+1}, u_{n+1}, y_{n+1} \mid x_{n}, u_{n}, y_{n}\right)= \\
& p\left(x_{n+1} \mid x_{n}, u_{n}\right) p\left(u_{n+1} \mid x_{n}, u_{n}\right) p\left(y_{n+1} \mid x_{n+1}\right)
\end{aligned}
$$

with $p\left(x_{n+1} \mid x_{n}, u_{n}\right), p\left(u_{n+1} \mid x_{n}, u_{n}\right)$, and $p\left(y_{n+1} \mid x_{n+1}\right)$ given by (2), (3), and (4), respectively. Then the "backward" probabilities $\beta_{i}\left(x_{i}, u_{i}\right)=p\left(y_{i+1}, \ldots y_{n} \mid x_{i}, u_{i}\right)$, needed to different useful computations, are recursively calculated by

$$
\begin{aligned}
& \beta_{n}\left(x_{n}, u_{n}\right)=1, \text { and } \beta_{i}\left(x_{i}, u_{i}\right)= \\
& \sum_{u_{i+1}, x_{i+1}} \beta_{i+1}\left(x_{i+1}, u_{i+1}\right) p\left(x_{i+1} \mid x_{i}, u_{i}\right) p\left(u_{i+1} \mid x_{i}, u_{i}\right) p\left(y_{i+1} \mid x_{i+1}\right)
\end{aligned}
$$

for $1 \leq i \leq n-1$

Of course, the sum in (6) is particular because of (2) and (3).

In order to simplify notations, let us put $V=(X, U)$. Thus $v_{i}=\left(x_{i}, u_{i}\right)$ for each $1 \leq i \leq n$. In particular, (6) is written

$$
\begin{aligned}
& \beta_{n}\left(v_{n}\right)=1, \text { and } \\
& \beta_{i}\left(v_{i}\right)=\sum_{v_{i+1}} \beta_{i+1}\left(v_{i+1}\right) p\left(v_{i+1} \mid v_{i}\right) p\left(y_{i+1} \mid v_{i+1}\right)
\end{aligned}
$$

for $1 \leq i \leq n-1$

(7) Looks like the formulas of a very classical hidden Markov chain $(V, Y)$; however, let us remark that the distributions $p\left(y_{i+1} \mid v_{i+1}\right)=p\left(y_{i+1} \mid x_{i+1}, u_{i+1}\right)$ verify

$$
p\left(y_{i+1} \mid x_{i+1}, u_{i+1}\right)=p\left(y_{i+1} \mid x_{i+1}\right)
$$

which means that a same noise distribution can remain valid for different classes $v_{i+1}$, which is not usual in classical models.

However, once it has been noticed that $(V, Y)$ is a hidden Markov chain, the "forward" probabilities $\alpha_{i}\left(v_{i}\right)=p\left(v_{i}, y_{1}, \ldots, y_{i}\right)$ can be computed recursively by

$$
\begin{aligned}
& \alpha_{i}\left(v_{1}\right)=p\left(v_{1}, y_{1}\right), \text { and } \\
& a_{i+1}\left(v_{+i}\right)=\sum_{v_{i}} \alpha_{i}\left(v_{i}\right) p\left(v_{i+1} \mid v_{i}\right) p\left(y_{i+1} \mid v_{i+1}\right)
\end{aligned}
$$

for $1+1 \leq i \leq n$.

Finally, backward and forward probabilities can be classically used to calculate: 


$$
\begin{aligned}
& p\left(v_{i+1} \mid v_{i}, y\right)=\frac{\beta_{i+1}\left(v_{i+1}\right)}{\beta_{i}\left(v_{i}\right)} \\
& p\left(v_{i} \mid y\right)=\frac{\alpha_{i}\left(v_{i}\right) \beta_{i}\left(v_{i}\right)}{\sum_{v_{i}^{\prime}} \alpha_{i}\left(v_{i}^{\prime}\right) \beta_{i}\left(v_{i}^{\prime}\right)} \\
& p\left(v_{i}, v_{i+1} \mid y\right)=\frac{\alpha_{i}\left(v_{i}\right) p\left(v_{i+1} \mid v_{i}\right) p\left(y_{i+1} \mid v_{i+1}\right) \beta_{i+1}\left(v_{i+1}\right)}{\sum_{v_{i}^{\prime}, v_{i+1}^{\prime}} \alpha_{i}\left(v_{i}\right) p\left(v_{i+1} \mid v_{i}\right) p\left(y_{i+1} \mid v_{i+1}\right) \beta_{i+1}\left(v_{i+1}\right)}
\end{aligned}
$$

Therefore we have a first TMC $T=(X, U, Y)$ which models the fact that $(X, Y)$ is an HSMC-IN.

\section{Remark 1}

Let us briefly mention that, as described in [11], each equation among (2)-(4) can be extended and thus the present viewpoint representing HSMC-IN as particular TMC enables one to propose numerous generalizations of HSMC-IN. For example, let us replace in equation (4) $p\left(y_{n+1} \mid x_{n+1}\right)$ by $p\left(y_{n+1} \mid u_{n}, x_{n+1}\right)$. Such an extension means that the distribution of the noise at $n+1$ also depends on the residual sojourn duration $u_{n}$. This can be understood intuitively; in fact, when this duration is large we are far away from the boundary among two different classes, and when $u_{n}=1$, we know that the class has just changed $\left(x_{n+1} \neq x_{n}\right)$. Thus replacing $p\left(y_{n+1} \mid x_{n+1}\right)$ by $p\left(y_{n+1} \mid u_{n}, x_{n+1}\right)$ enables one to model the fact that on boundaries the distribution of the noise can be different from its distribution "inside" of a given class. Then the modelling of the non stationarity of $X$ proposed in this paper can still be extended to these different generalizations of the HSMC-IN model.

\section{HIDDEN EVIDENTIAL MARKOV CHAIN}

Let us consider the HMC-IN $T=(V, Y)$ above and let us consider that this HMC-IN is not stationary. In other words, the distribution $p\left(v_{i}, y_{i}, v_{i+1}, y_{i+1}\right)$ depends on $1 \leq i \leq n-1$. Furthermore, let us assume that this non stationarity is due to the non-stationarity of $V$, which means that $p\left(y_{i} \mid v_{i}\right)$ does not depend on $1 \leq i \leq n$. Such a situation has been studied in [8] by the use of DempsterShafer theory of evidence. By replacing the non stationary prior distribution of $V$ by evidential priors as explained in $[7,8]$, we necessarily replace the non stationary distribution of $V=(X, U)$ by some particular evidential priors. As we are going to specify, such a replacement amounts to introducing in the HMC-IN $(V, Y)$ a third random chain $V^{\prime}=\left(V_{1}^{\prime}, \ldots, V_{n}^{\prime}\right)$, where each $V_{i}^{\prime}$ takes its values in a finite set $\Delta^{\prime}=\left\{\lambda_{1}, \ldots, \lambda_{m}\right\}$, and leads to the consideration of the TMC $\left(V, V^{\prime}, Y\right)$. Finally a nonstationary HMC-IN $(V, Y)$ will be replaced by a stationary TMC $\left(V, V^{\prime}, Y\right)$.

Let us specify with some more details the use of the theory of evidence. Each $V_{i}$ taking its values in $\Delta=\left\{\delta_{1} \ldots, \delta_{r}\right\}$, let us denote by $[\mathrm{P}(\Delta)]$ the power set of $\Delta$. We will consider the so-called "evidential Markov chain" (EMC), denoted by $m$, which verifies:

(i) $m$ is defined on $[\mathrm{P}(\Delta)]^{n}$ and takes its values in $[0,1]$;

(ii) $\sum_{A \in[\mathrm{P}(\Delta)]^{n}} m(A)=1$ and $m\left(A_{1} \times \ldots \times A_{n}\right)=0$ if one at least among $A_{1}, \ldots, A_{n}$ is $\varnothing$;

(iii) $m$ is of the "Markovian" form : $m\left(A_{1}, A_{2}, \ldots, A_{n}\right)=$ $m\left(A_{1}\right) m\left(A_{2} \mid A_{1}\right) \ldots m\left(A_{n} \mid A_{n-1}\right)$.

We see how an EMC extends a Markov chain; in fact, when $m\left(A_{1} \times \ldots \times A_{n}\right)=0$ if one at least among $A_{1}, \ldots, A_{n}$ is not a singleton, $m$ is a Markov chain on $\Delta^{n}$.

The approach proposed in [8] is based on the two following points:

1. The posterior distribution $p(v \mid y)$ of an HMC-IN, which is needed to Bayesian restoration, can be seen as the DS fusion of the prior Markov distribution $p(v)=p\left(v_{1}\right) p\left(v_{2} \mid v_{1}\right) \ldots p\left(v_{n} \mid v_{n-1}\right) \quad$ of $\quad V \quad$ with the probability $\quad q^{y}\left(v_{1}, \ldots, v_{n}\right) \propto p\left(y_{1} \mid v_{1}\right) \ldots p\left(y_{n} \mid v_{n}\right) \quad$ (where “ $\propto$ " means "proportional to") defined on $\Delta^{n}$ by $Y=y$;

2. When the distribution $p(v)$ of the Markov chain $V$ is incompletely known, it can be replaced by an EMC obtained from $p(v)$, whose aim is to model the lack of precise knowledge of $p(v)$. It can be fused with $q^{y}$ using DS fusion. The result of the latter fusion is a probability distribution on $\Delta^{n}$ and, although it is not necessarily a Markov distribution, it can be used to perform Bayesian restorations. Indeed, the latter feasibility is due to the fact that the fused distribution is a triplet Markov chain [8]. To be more precise, $p(v)$ is replaced by an EMC $m$ such that $m\left(A_{1} \times \ldots \times A_{n}\right) \neq 0$ only on $\left(A_{1} \times \ldots \times A_{n}\right)$ such that each $A_{i}$ is in $\Delta^{\prime}=\left\{\left\{\delta_{1}\right\} \ldots,\left\{\delta_{r}\right\}, \Delta\right\}$. Thus a homogeneous Markov chain on $\Delta^{n}$ is defined by $r^{2}$ parameters, and a 
homogeneous EMC on $\left\{\left\{\delta_{1}\right\} \ldots,\left\{\delta_{r}\right\}, \Delta\right\}$ is defined by $(r+1)^{2}$ parameters, which means that EMC considered here is not so much more complex than the corresponding Markov chain.

Finally, let us specify how the DS fusion (DS fusion) allows one to calculate different quantities of interest.

Let us consider the DS fusion of the probability $q^{y}\left(v_{1}, \ldots, v_{n}\right) \propto p\left(y_{1} \mid v_{1}\right) \ldots p\left(y_{n} \mid v_{n}\right) \quad$ with $\quad m\left(A_{1} \times \ldots \times A_{n}\right)$ defined above. We have:

$\left(q^{y} \oplus m\right)\left(v_{1}, \ldots, v_{n}\right) \propto$

$$
\sum_{v_{n} \in\left(v_{1}^{\prime}, \ldots, v_{n}^{n}\right)} q^{y}\left(v_{1}, \ldots, v_{n}\right) m\left(v_{1}^{\prime}, \ldots, v_{n}^{\prime}\right)
$$

where each $v_{i}$ is in $\Delta=\left\{\delta_{1} \ldots, \delta_{r}\right\}$, each $v_{i}^{\prime}$ is in $\Delta^{\prime}=\left\{\left\{\delta_{1}\right\} \ldots,\left\{\delta_{r}\right\}, \Delta\right\}$, and $\left(v_{1}, \ldots, v_{n}\right) \in\left(v_{1}^{\prime}, \ldots, v_{n}^{\prime}\right)$ means that $v_{1} \in v_{1}^{\prime}, \ldots, \quad v_{n} \in v^{\prime}$. Let us insist on the fact that in (13) $\left(v_{1}, \ldots, v_{n}\right)$ and the sum is taken over $\left(v_{1}^{\prime}, \ldots, v_{n}^{\prime}\right)$ such that $\left(v_{1}, \ldots, v_{n}\right) \in\left(v_{1}^{\prime}, \ldots, v_{n}^{\prime}\right)$.

Important is then to notice that $\left(v_{1}, \ldots, v_{n}\right) m\left(v_{1}^{\prime}, \ldots, v_{n}^{\prime}\right)$ in (13) defines a Markov chain, and the DS fusion is the calculation of some marginal distribution of this Markov chain.

To be more precise, let us consider $n-1$ functions $f_{2}^{y_{1}, y_{2}}$, $f_{3}^{y_{3}}, \ldots, f_{n}^{y_{n}}$ defined on $\Delta^{2} \times\left(\Delta^{\prime}\right)^{2}$ by:

$$
\begin{aligned}
& f_{2}^{y_{1}, y_{2}}\left(v_{1}, v_{2}, v_{1}^{\prime}, v_{2}^{\prime}\right)= \\
& 1_{\left[v_{1} \in v_{1}^{\prime}, v_{2} \in v_{v^{\prime}}\right]} p\left(y_{1} \mid v_{1}\right) p\left(y_{2} \mid v_{2}\right) m\left(v_{1}^{\prime}, v_{2}^{\prime}\right)
\end{aligned}
$$

and

$$
\begin{aligned}
& f_{i+1}^{y_{i+1}}\left(v_{i}, v_{i+1}, v_{i}^{\prime}, v_{i+1}^{\prime} y_{i+1}\right)= \\
& 1_{\left[v_{i} \in v_{i}^{\prime}, v_{i+1} \in v_{i+1}^{\prime}\right]} p\left(y_{i+1} \mid v_{i+1}\right) m\left(v_{i+1}^{\prime} \mid v_{i}^{\prime}\right)
\end{aligned}
$$

for $2 \leq i \leq n-1$.

On the one hand, the product $f_{2}^{y_{1}, y_{2}}\left(v_{1}, v_{2}, v_{1}^{\prime}, v_{2}^{\prime}\right) \times$ $f_{3}^{y_{3}}\left(v_{2}, v_{3}, v_{2}^{\prime}, v_{3}^{\prime}\right) \times \ldots \times f_{n}^{y_{3}}\left(v_{n-1}, v_{n}, v_{n-1}^{\prime}, v_{n}^{\prime}\right)$ defines (for fixed $y)$ a Markov chain on $\Delta^{n} \times\left(\Delta^{\prime}\right)^{n}$ with calculable $p\left(v_{i+1}, v_{i+1}^{\prime} \mid v_{i}, v_{i}^{\prime}, y\right), p\left(v_{i}, v_{i}^{\prime}, v_{i+1}, v_{i+1}^{\prime} \mid y\right)$ and $p\left(v_{i}, v_{i}^{\prime} \mid y\right)$ (see Lemma below). On the other hand, we see that this product is proportional to $q^{y}\left(v_{1}, \ldots, v_{n}\right) m\left(v_{1}^{\prime}, \ldots, v_{n}^{\prime}\right)$ in (13). These two results show that the result of the DS fusion $\left(q^{y} \oplus m\right)\left(v_{1}, \ldots, v_{n}\right)$ in (13) is the marginal distribution (the distribution of $\left.\left(V_{1}, \ldots, V_{n}\right)\right)$ of the distribution of the Markov chain $\left(\left(V_{1}, V_{1}^{\prime}\right), \ldots,\left(V_{n}, V_{n}^{\prime}\right)\right)$ defined by the functions $f_{2}^{y_{1}, y_{2}}, f_{3}^{y_{3}}, \ldots, f_{n}^{y_{n}}$. Finally, we can state the following result:

\section{Proposition 1}

Let $(V, Y)=\left(V_{1}, Y_{1}, \ldots, V_{n}, Y_{n}\right)$ be a classical HMC-IN, each $V_{i}$ taking its values in $\Delta=\left\{\delta_{1} \ldots, \delta_{r}\right\}$ and each $Y_{i}$ taking its values in $R$. Therefore, $p(v)=p\left(v_{1}\right) p\left(v_{2} \mid v_{1}\right) \ldots p\left(v_{n} \mid v_{n-1}\right), \quad$ and $p(y \mid v)=p\left(y_{1} \mid v_{1}\right) \ldots p\left(y_{n} \mid v_{n}\right)$.

Let $q^{y}\left(v_{1}, \ldots, v_{n}\right) \propto p\left(y_{1} \mid v_{1}\right) \ldots p\left(y_{n} \mid v_{n}\right)$ be the probability defined on $\Delta^{n}$ by $Y=y$, and let $m$ be an EMC on $\Delta^{\prime}=\left\{\left\{\delta_{1}\right\} \ldots,\left\{\delta_{r}\right\}, \Delta\right\}$ extending the prior distribution $p(v)=p\left(v_{1}\right) p\left(v_{2} \mid v_{1}\right) \ldots p\left(v_{n} \mid v_{n-1}\right)$.

Then the probability $q^{y} \oplus m$ given by (13), which extends the posterior probability $p(v \mid y)$ and which is not necessarily of Markovian form, is a marginal probability of a finite Markov chain. As a consequence, $q^{y} \oplus m$ can be used to perform different restorations like MPM.

This result has been successfully applied to the case of non-stationary hidden $V=\left(V_{1}, \ldots, V_{n}\right)$, see [8].

\section{HIDDEN EVIDENTIAL SEMI-MARKOV CHAIN}

Let us return to the situation of the previous section, with the hidden semi-Markov chain $X=\left(X_{1}, \ldots, X_{n}\right)$, $Y=\left(Y_{1}, \ldots, Y_{n}\right)$, where each $X_{i}$ takes its values in $\Omega=\left\{\omega_{1}, \ldots, \omega_{k}\right\}$ and each $Y_{i}$ takes its values in $R$. As specified above, the HSMC-IN $(X, Y)$ can also be seen as a TMC $(X, U, Y)$, which also is an $\operatorname{HMC-IN}(V, Y)$, with $V=(X, U)$. Let us consider the case of non-stationary semi-Markov chain $X=\left(X_{1}, \ldots, X_{n}\right)$, which means that $V=(X, U)$ also is non stationary. Thus this nonstationarity of $V=(X, U)$ can be managed as specified in Proposition 1. Knowing that $p(v \mid y)$ is workable as being marginal distribution of the Markov chain $p\left(v, v^{\prime} \mid y\right)$, we see that $p(x \mid y)$ is workable as being a marginal distribution of $p(v \mid y)=p(x, u \mid y)$, and thus a marginal distribution of the Markov chain $p\left(x, u, v^{\prime} \mid y\right)$. As an example, let us detail how the posterior marginal 
distributions $p\left(x_{i} \mid y\right)$, enabling one to perform the MPM segmentation, are computable.

\section{Proposition 2}

Let $X$ be a non-stationary semi-Markov chain, $(X, Y)$ a non-stationary HSMC-IN, $(X, U, Y)$ the corresponding non-stationary TMC, and $\left(X, U, V^{\prime}, Y\right)$ a stationary Markov chain obtained from $(X, U, Y)=(V, Y)$ as specified above.

Then $p\left(x_{i} \mid y\right)$ is computable in two steps:

(i) $p\left(x_{i}, u_{i} \mid y\right)=p\left(v_{i}, v_{i}^{\prime} \mid y\right)=\sum_{v_{i}^{\prime}} p\left(v_{i}, v_{i}^{\prime} \mid y\right)$, and

(ii) $p\left(x_{i} \mid y\right)=\sum_{u_{i}} p\left(x_{i}, u_{i} \mid y\right)$.

Finally, we arrived at a TMC $\left(X, U, V^{\prime}, Y\right)=(X, W, Y)$, where the third random process $W=\left(U, V^{\prime}\right)$ model the fact that $X$ is semi-Markov on the one hand, and the fact that $X$ is non-stationary, on the other hand.

\section{Remark 2}

We pointed out in Remark 1 above how the model $T=\left(X, U, V^{\prime}, Y\right)$ can be extended by considering different extensions offered by (2)-(5). However, all over the paper we kept the notations "HMC-IN" and HSMC-IN" to clearly specify that in all models considered the random variables $Y_{1}, \ldots, Y_{n}$ remain independent conditionally on $X$, which means that the "noise" is of a quite simple and undoubtedly too simple in numerous situations - form. However, extending this simple case to correlated noise is quite straightforward in the Gaussian noise case. When the noise is correlated and not necessarily Gaussian, it is then possible to use the theory of Copulas, as proposed in the simple HMC-IN case in [3].

\section{Lemma}

Let $H=\left(H_{1}, \ldots, H_{n}\right)$ be a random chain, each $H_{i}$ taking its values in a finite set.

Then $H$ is a Markov chain if and only if there exist positive functions $s_{1}, \ldots, \quad s_{n-1}$ such that $p\left(h_{1}, \ldots, h_{n}\right) \propto s_{1}\left(h_{1}, h_{2}\right) \ldots s_{n-1}\left(h_{n-1}, h_{n}\right)$.

If $H$ is a Markov chain, the transitions and the marginal distributions are given by

$$
\begin{aligned}
& p\left(h_{i+1} \mid h_{i}\right)=q_{i+1}\left(h_{i}, h_{i+1}\right) \beta_{i+1}\left(h_{i+1}\right) / \beta_{i}\left(h_{i}\right), \\
& p\left(h_{i}\right)=\alpha_{i}\left(h_{i}\right) \beta_{i}\left(h_{i}\right) / \sum_{h_{i}^{\prime}} \alpha_{i}\left(h_{i}^{\prime}\right) \beta_{i}\left(h_{i}^{\prime}\right),
\end{aligned}
$$

with

$$
\begin{aligned}
& \alpha_{1}\left(h_{1}\right)=1, \alpha_{i+1}\left(h_{i+1}\right)=\sum_{h_{i+1}} q_{i+1}\left(h_{i}, h_{i+1}\right) \alpha_{i}\left(h_{i}\right), \text { and } \\
& \beta_{n}\left(h_{n}\right)=1, \beta_{i}\left(h_{i}\right)=\sum_{h_{i+1}} q_{i+1}\left(h_{i}, h_{i+1}\right) \beta_{i+1}\left(h_{i+1}\right) .
\end{aligned}
$$

\section{PARAMETER ESTIMATION}

Let us briefly mention how the parameter estimation can be performed by two general methods 'ExpectationMaximization" (EM), and 'Iterative Conditional Estimation" (ICE). To simplify things, let us assume that the variables $U_{i}$ take their values in a finite set. Moreover, we assume that the TMC $T=\left(V, V^{\prime}, Y\right)$ is "Gaussian" in that $p\left(y \mid v, v^{\prime}\right)$ are Gaussian. Assuming $T$ stationary in that neither $p\left(v_{i}, v_{i}^{\prime}, v_{i+1}, v_{i+1}^{\prime}\right)$ nor $p\left(y_{i} \mid v_{i}, v_{i}^{\prime}\right)=p\left(y_{i} \mid x_{i}\right)$ depend on $i$, we have to estimate the finite distribution $p\left(v_{i}, v_{i}^{\prime}, v_{i+1}, v_{i+1}^{\prime}\right)$ on $\Delta^{2} \times\left(\Delta^{\prime}\right)^{2}$, and, for $k$ classes, $k$ means and $k$ variances of the $k$ Gaussian distributions $p\left(y_{i} \mid x_{i}\right)$ on $R$. Let us put $\Delta^{2} \times\left(\Delta^{\prime}\right)^{2}=\{1, \ldots, M\}$, $\tau_{j}=p\left[\left(v_{i}, v_{i}^{\prime}, v_{i+1}, v_{i+1}^{\prime}\right)=j\right] \quad$ and $\quad \tau=\left(\tau_{1}, \ldots, \tau_{M}\right)$. Otherwise, let $\mu=\left(\mu_{1}, \ldots, \mu_{k}\right)$ be the means and $\sigma^{2}=\left(\sigma_{1}^{2}, \ldots, \sigma_{k}^{2}\right)$ the variances. Then the parameters to be estimated are $\theta=\left(\tau, \mu, \sigma^{2}\right)$. Both EM and ICE methods are iterative: taking an initial value $\theta^{0}$, the problem is to compute $\theta^{m+1}$ from the observation $Y=y=\left(y_{1}, \ldots, y_{n}\right)$ and $\theta^{m}$

EM method runs as follows:

(i) use $\theta^{m}$ to calculate $\tau_{j}^{i, m}=p\left[\left(v_{i}, v_{i}^{\prime}, v_{i+1}, v_{i+1}^{\prime}\right)=j \mid y, \theta^{m}\right]$ (using the Lemma) and $p_{q}^{i, m}=p\left[x_{i}=\omega_{q} \mid y, \theta^{m}\right]$ (deduced from $\left.\tau_{j}^{i, m}\right)$;

(ii) put $\tau_{j}^{m+1}=\frac{\tau_{j}^{1, m}+. . \tau_{j}^{n-1, m}}{n-1} \quad$ for $\quad 1 \leq j \leq M$, $\mu_{q}^{m+1}=\frac{y_{1} p_{q}^{1, m}+\ldots+y_{n} p_{q}^{n, m}}{p_{q}^{1, m}+\ldots+p_{q}^{n, m}}$, and $\sigma_{q}^{2, m+1}=\frac{\left(y_{1}-\mu_{q}^{m+1}\right)^{2} p_{q}^{1, m}+\ldots+\left(y_{n}-\mu_{q}^{m+1}\right)^{2} p_{q}^{n, m}}{p_{q}^{1, m}+\ldots+p_{q}^{n, m}}$ for $1 \leq q \leq k$, which gives $\theta^{m+1}$.

In ICE $\tau_{j}^{m+1}$ is obtained as in EM and, to obtain $\mu_{q}^{m+1}$ and $\sigma_{q}^{2, m+1}$, one simulates $a$ realisations $x^{1}=\left(x_{1}^{1}, \ldots, x_{n}^{1}\right), \ldots$, $x^{a}=\left(x_{1}^{a}, \ldots, x_{n}^{a}\right) \quad$ of $\quad X=\left(X_{1}, \ldots, X_{n}\right) \quad$ according to $p\left(x \mid y, \theta^{m}\right) \quad$ (recall that the distribution of $\left(V, V^{\prime}\right)=\left(X, U, V^{\prime}\right)$ conditional on $Y$ is a Markov 
distribution with computable transitions $p\left(v_{i+1}, v_{i+1}^{\prime} \mid v_{i}, v_{i}^{\prime}, y, \theta^{m}\right), \quad$ and thus simulating $\left(V, V^{\prime}\right)=\left(X, U, V^{\prime}\right)$ gives $\left.X=x\right)$. For $1 \leq l \leq a$ and $1 \leq q \leq k$, let $x^{l, q}=\left(x_{1}^{l, q}, \ldots, x_{n^{\prime}}^{l, q}\right)$ be the sub-sample of $x^{l}=\left(x_{1}^{l}, \ldots, x_{n}^{l}\right)$ such that $x_{1}^{l, q}=\omega_{q}, \ldots, \quad x_{n^{\prime}}^{l, q}=\omega_{q}$. This sub-sample is then used to estimate, by the classical estimators, the mean $\mu_{q}^{m+1, l}$, and the variance $\sigma_{q}^{2, m+1, l}$. Then $\mu_{q}^{m+1}=\frac{\mu_{q}^{m+1,1}+\ldots+\mu_{q}^{m+1, a}}{a}$, and $\sigma_{q}^{2, m+1}=\frac{\sigma_{q}^{2, m+1,1}+\ldots \sigma_{q}^{2, m+1, a}}{a}$. In practice, one often takes $a=1$.

\section{CONCLUSION AND PERSPECTIVES}

We dealt in this paper with unsupervised segmentation of the hidden non stationary semi-Markov chains. The main tool used was the triplet Markov chain model, which has been obtained by introduction of two auxiliary chains. The first auxiliary chain modelled the semi-Markovianity, and the second one modelled the non stationarity.

As perspectives, we can mention different possibilities of further extensions. In particular, more complex noises can be introduced via the Copula theory, as described in the simple hidden Markov chains case in [3]. Otherwise, the mono sensor case considered in this paper (observations in $R$ ) can be extended to the multisensor one [12].

\section{REFERENCES}

[1] B. Ait-el-Fquih and F. Desbouvries, Kalman filtering for triplet Markov chains : applications and extensions, Proceedings of the International Conference on Acoustics, Speech and Signal Processing (ICASSP 05), March 19-23, Philadelphia, USA, 2005.

[2] A. Appriou, Probabilités et incertitude en fusion de données multisenseurs, Revue Scientifique et Technique de la Défense, No. 11, pp. 27-40, 1991.

[3] N. Brunel and W. Pieczynski, Unsupervised signal restoration using Copulas and Pairwise Markov chains, IEEE Workshop on Statistical Signal Processing (SSP 2003), Saint Louis, Missouri, September 28-October 1, 2003.

[4] F. Desbouvries and W. Pieczynski, Particle Filtering in Pairwise and Triplet Markov Chains, Proceedings of the IEEE - EURASIP Workshop on Nonlinear Signal and Image Processing (NSIP 2003), Grado-Gorizia, Italy, June 8-11, 2003.
[5] S. Faisan, L. Thoraval, J.-P. Armspach, F. Heitz, Hidden Semi-Markov Event Sequence Models: Application to Brain Functional MRI Sequence Analysis, IEEE Int. Conf. Image Processing, ICIP 2002, Rochester, USA, Sept. 2002.

[6] Y. Guedon and C. Cocozza-Thivent, Use of the Derin' s algorithm in hidden semi-Markov models for automatic speech recognition, International Conference on Acoustics, Speech, and Signal Processing, pp. 282-285, 1989.

[7] P. Lanchantin et W. Pieczynski, Chaînes et arbres de Markov évidentiels avec applications à la segmentation des processus non stationnaires, Traitement du Signal, Vol. 22, No. 1, 2005.

[8] P. Lanchantin and W. Pieczynski, Unsupervised restoration of hidden non stationary Markov chain using evidential priors, IEEE Trans. on Signal Processing, Vol. 53, No. 8, 2005.

[9] W. Pieczynski, Pairwise Markov chains, IEEE Transactions on Pattern Analysis and Machine Intelligence, Vol. 25, No. 5, pp. 634-639, 2003.

[10] W. Pieczynski, C. Hulard, and T. Veit, Triplet Markov chains in hidden signal restoration, SPIE's International Symposium on Remote Sensing, September 22-27, Crete, Greece, 2002.

[11] W. Pieczynski, Chaînes Chaînes semi-markoviennes cachées et chaînes de Markov triplet, soumis à Comptes Rendus de l'Académie des Sciences - Mathématique, décembre 2004 (in French).

[12] W. Pieczynski, Fusion de Dempster-Shafer dans les chaînes triplet partiellement de Markov, Comptes Rendus de l'Académie des Sciences - Mathématique, Série I, Vol. 339, Issue 11, pp. 797-802, 2004.

[13] W. Pieczynski and F. Desbouvries, On triplet Markov chains, International Symposium on Applied Stochastic Models and Data Analysis, (ASMDA 2005), Brest, France, May 2005.

[14] G. Shafer, A mathematical theory of evidence, Princeton University Press, Princeton, 1976.

[15] S.-Z. Yu and H. Kobayashi, A hidden semi-Markov model with missing data and multiple observation sequences for mobility tracking, Signal Processing, Vol. 83, No. 2, pp. 235-250, 2003. 